\title{
Chi-square test on candidate events from CW signal coherent searches
}

\author{
Y Itoh ${ }^{1}$, M A Papa ${ }^{1}$, B Krishnan ${ }^{1}$ and X Siemens ${ }^{2}$ \\ ${ }^{1}$ Max Planck Institut für Gravitationsphysik, Albert Einstein Institut, Am Mühlenberg 1, \\ Golm 14476, Germany \\ 2 Center for Gravitation and Cosmology, Department of Physics, \\ University of Wisconsin-Milwaukee, PO Box 413, WI 53201, USA \\ E-mail: yousuke.itoh@aei.mpg.de
}

Received 7 May 2004

Published 24 September 2004

Online at stacks.iop.org/CQG/21/S1667

doi:10.1088/0264-9381/21/20/009

\begin{abstract}
In a blind search for continuous gravitational wave signals scanning a wide frequency band one looks for candidate events with significantly large values of the detection statistic. Unfortunately, a noise line in the data may also produce a moderately large detection statistic. In this paper, we describe how we can distinguish between noise line events and actual continuous wave (CW) signals, based on the shape of the detection statistic as a function of the signal's frequency. We will analyse the case of a particular detection statistic, the $F$ statistic, proposed by Jaranowski, Królak and Schutz. We will show that for a broad-band $10 \mathrm{~h}$ search, with a false dismissal rate smaller than $10^{-6}$, our method rejects about $70 \%$ of the large candidate events found in a typical data set from the second science run of the Hanford LIGO interferometer.
\end{abstract}

PACS numbers: $04.80 . \mathrm{Nn}, 95.75 .-\mathrm{Z}$

(Some figures in this article are in colour only in the electronic version)

\section{Introduction}

High power in a narrow frequency band (spectral lines) are common features of an interferometric gravitational wave (GW) detector's output. Although continuous gravitational waves could show up as lines in the frequency domain, given the current sensitivity of GW detectors it is most likely that large spectral features are noise of terrestrial origin or statistical fluctuations.

Monochromatic signals of extraterrestrial origin are subject to a Doppler modulation due to the detector's relative motion with respect to the extraterrestrial GW source, while those of terrestrial origin are not. Matched filtering techniques to search for a monochromatic 
signal from a given direction in the sky demodulate the data based on the expected frequency modulation from a source in that particular direction. In general, this demodulation procedure decreases the significance of a noise line and enhances that of a real signal. However, if the noise artefact is large enough, even after the demodulation it might still present itself as a statistically significant outlier, thus a candidate event. Our idea to discriminate between an extraterrestrial signal and a noise line is based on the different effect that the demodulation procedure has on a real signal and on a spurious one.

If the data actually contain a signal, the detection statistic presents a very particular pattern around the signal frequency which, in general, a random noise artefact does not. We propose here a chi-square test based on the shape of the detection statistic as a function of the signal frequency and demonstrate its safety and its efficiency. We use the $F$ detection statistic described in [1] and adopt the same notation as [1]. For applications of the $F$ statistic search on real data, see for example [2-4].

\section{Method}

\subsection{Summary of the method}

In this paper, we consider a continuous GW signal such as we would expect from an isolated non-axisymmetric rotating neutron star. Following the notation of [1], the parameters that describe such a signal are its emission frequency $f_{s}$, the position in the sky of the source $\vec{l}_{s}=\left(\alpha_{s}, \delta_{s}\right)$, the amplitude of the signal $h_{0}$, the inclination angle $\iota$, the polarization angle $\psi$ and the initial phase of the signal $2 \Phi_{0}$.

In the absence of a signal $2 F$ follows a $\chi^{2}$ distribution with four degrees of freedom (which will be denoted by $\chi_{4}^{2}$ ). In the presence of a signal $2 F$ follows a non-central $\chi_{4}^{2}$ distribution.

Given a set of template parameters $(\vec{l}, f)$, the detection statistic $F$ is the likelihood function maximized with respect to the parameters $\vec{p}_{s}=\left(h_{0}, \iota, \psi, \Phi_{0}\right) . F$ is constructed by combining appropriately the complex amplitudes $F_{a}$ and $F_{b}$ representing the complex matched filters for the two GW polarizations. And given the template parameters and the values of $F_{a}$ and $F_{b}$ it is possible to derive the maximum likelihood values of $\left(h_{0}, \iota, \psi, \Phi_{0}\right)$-let us refer to these as $\vec{p}_{\text {MLE }}$. It is thus possible for every value of the detection statistic to estimate the parameters of the signal that have most likely generated it. So, if we detect a large outlier in $F$ we can estimate the associated signal parameters: $\left(\vec{l}, f, \vec{p}_{\mathrm{MLE}}\right)$. Let us indicate with $\tilde{s}(t)$ the corresponding signal estimate.

Let $x(t)$ be the original data set, and define a second data set

$$
\tilde{x}(t) \equiv x(t)-\tilde{s}(t)
$$

If the outliers were actually due to a signal $s(t)$ and if $\tilde{s}(t)$ were a good approximation to $s(t)$, then $2 \tilde{F}$ constructed from $\tilde{x}(t)$ would be $\chi_{4}^{2}$ distributed.

Since filters for different values of $f$ are not orthogonal, in the presence of a signal the detection statistic $F$ also presents some structures for values of search frequency that are not the actual signal frequency. For these other frequencies $2 \tilde{F}$ is also $\chi_{4}^{2}$ distributed if $\tilde{s}(t)$ is a good approximation to $s(t)$.

We thus construct the veto statistic $\mathcal{V}$ by summing the values of $2 \tilde{F}$ over more frequencies. In particular, we sum over all the neighbouring frequency bins that, within a certain frequency interval, are above a fixed significance threshold. We regard each such collection of frequencies as a single 'candidate event' and assign to it the frequency of the bin that has the highest value of the detection statistic. The veto statistic is then

$$
\mathcal{V}:=\sum_{k \in \text { event }} 2 \tilde{F}\left(f_{k}\right)
$$


In reality, since our templates lie on a discrete grid, the parameters of a putative signal will not exactly match any templates' parameters and the signal estimate $\tilde{s}(t)$ will not be exactly correct. As a consequence $\tilde{x}(t)$ will still contain a residual signal and $\tilde{F}$ will not exactly be $\chi_{4}^{2}$ distributed. The larger the signal, the larger the residual signal and the larger the expected value of $\mathcal{V}$. Therefore, our veto threshold $\mathcal{V}_{\text {thr }}$ will not be fixed but will depend on the value of $F$. We will find such a $F$-dependent threshold for $\mathcal{V}$ based on Monte Carlo simulations. The signal-to-noise ratio (SNR) for any given value of the detection statistic can be expressed in terms of the detection statistic as $\sqrt{2 F}$, as per equation (79) of [1]. Therefore, we will talk equivalently of an SNR-dependent or $F$-dependent veto threshold.

\subsection{Stationary Gaussian noise plus a signal with exactly known parameters}

Let us first examine the ideal case where the detector output consists of stationary random Gaussian noise plus a systematic time series (a noise line or a pulsar signal) that produces a candidate in the detection statistic $F(f)$ for some template sky position $\vec{l}$ and at frequency $f$. The question that we want to answer is: is the shape of $F(f)$ around the frequency of the candidate consistent with what we would expect from a signal?

Our basic observables are the four real inner products $X_{i}(f, \vec{l})$ between the observed time series $x(t)$ and the four filters $h_{i}(t ; \vec{l}, f)$ :

$$
X_{i}(f, \vec{l})=\left(x(t) \| h_{i}(t ; \vec{l}, f)\right),
$$

where $i$ runs from 1 to 4 . The inner product is defined by equation (42) of [1]. The four filters $h_{i}(t ; \vec{l}, f)$ depend on the target frequency $f$ and the target sky location $\vec{l}=(\alpha, \delta)$.

The hypothesis $H_{0}$ that we would like to examine is

$$
H_{0}: x(t)=n(t)+s\left(t ; \vec{p}_{\mathrm{MLE}}, \vec{l}, f\right),
$$

where $n(t)$ is the detector noise and $s\left(t ; \vec{p}_{\mathrm{MLE}}, \vec{l}, f\right)=A_{i}\left(\vec{p}_{\mathrm{MLE}}\right) h_{i}(t ; \vec{l}, f)$ is the template, which in this case perfectly matches the signal. The parameters $\vec{p}_{\text {MLE }}$ are the maximum likelihood estimators of $h_{0}, \cos \iota, \psi, \Phi_{0}$ derived from the data and the template parameters $\vec{l}$ and $f$. The definitions of the four coefficients $A_{i}$ are given in [1].

Given that the template parameters $\vec{l}, f$ exactly match the parameters of the actual signal, then the waveform $\tilde{s}\left(t ; \vec{p}_{\text {MLE }}, \vec{l}, f\right)$ exactly matches the actual signal $s(t)$. In this case, the four variables $\tilde{X}_{i}(f, \vec{l})$ :

$$
\tilde{X}_{i}(f, \vec{l})=X_{i}(f, \vec{l})-\left(\tilde{s}(t) \| h_{i}(t ; \vec{l}, f)\right)
$$

are four correlated random Gaussian variables. The paper [1] constructs the detection statistic $F$ from the data $X_{i}(f)$. Similarly, we construct $\mathcal{F}_{v}(f):=F(f ; \tilde{X}(f))$ from the data $\tilde{X}_{i}(f)$. $\mathcal{F}_{v}(f)$ is also centrally $\chi_{4}^{2}$ distributed in the presence of a signal and perfect signal-template match. We obtain the veto statistic by summing $2 \mathcal{F}_{v}(f)$ over the different frequencies of the event

$$
\mathcal{V}:=\sum_{k=k_{1}, \ldots, k_{N} \in \text { event }} 2 \mathcal{F}_{v}\left(f_{k}\right)
$$

where $N$ is the number of the frequency bins in the event. If the value of $\mathcal{V}$ is not consistent with a $\chi_{4 N-4}^{2}$ distribution, we reject the hypothesis $H_{0}$. Note that the degrees of freedom of the veto statistic are $4 N-4$, as we use four data points to infer the four parameters $\vec{p}_{s}$.

\subsection{Real noise plus a signal with parameters mismatched with respect to the template}

In the real analysis, the signal parameters $\vec{l}_{s}$ will not exactly match the values of one of our templates $\vec{l}$. As a consequence, $\vec{p}_{\text {MLE }}$ will not match exactly the actual $\vec{p}_{s}$ parameters and the 
frequency where the maximum of the detection statistic occurs, $f_{\max }$, will not be the actual frequency of the signal $f_{s}$. However, we can still set up a procedure to answer the question: is the shape of the $F$ statistic event consistent with what we would expect from a signal with parameters close to $\vec{l}$ ?

Suppose that an event has been identified for a position template $\vec{l}$ and for a value of the signal frequency $f_{\max }$. This is how the veto analysis would proceed:

1. We determine $\vec{p}_{\text {MLE }}$ and $X_{i}\left(f_{k}, \vec{l}\right)$ for each $f_{k}$ of the event.

2. We generate a veto signal $\tilde{s}\left(f_{k} ; \vec{p}_{\text {MLE }}, \vec{l}, f_{\max }\right)$ and compute the four variables $S_{i}\left(f_{k}, \vec{l}\right)=$ $\left(\tilde{s}\left(t ; \vec{p}_{\mathrm{MLE}}, \vec{l}, f_{\max }\right) \| h_{i}\left(t ; \vec{l}, f_{k}\right)\right)$.

3. We construct the variables:

$$
\tilde{X}_{i}\left(f_{k}, \vec{l}\right)=X_{i}\left(f_{k}, \vec{l}\right)-S_{i}\left(f_{k}, \vec{l}\right) .
$$

4. Using equation (6) we compute $\mathcal{F}_{v}(f)$ and then $\mathcal{V}$.

If $\tilde{s}\left(f_{k} ; \vec{p}_{\text {MLE }}, \vec{l}, f_{\max }\right)$ is a good approximation to $s\left(f_{k} ; \vec{p}_{s}, \vec{l}_{s}, f_{s}\right)$, then $\mathcal{V}$ follows the $\chi_{4 N-4}^{2}$ distribution.

\subsection{SNR-dependent veto threshold}

As already outlined at the end of section 2.1, the veto statistic does not in general follow a $\chi_{4 N-4}^{2}$ distribution because in general the signal parameters do not exactly match the template parameters. Due to this mismatch when step 3 is performed in the procedure described in the previous section, not all the signals are removed from $X_{i}$. Consequently $\mathcal{V}$ acquires a nonzero centrality parameter. Since this scales as $h_{0}^{2}$ in the presence of a signal, the veto statistic threshold has to change with the SNR of the candidate event in order to keep the false dismissal rate constant for a range of different signal strengths. We will thus adopt an SNR-dependent veto threshold on our veto statistic $\mathcal{V}$. We will determine the threshold $\mathcal{V}_{\text {thr }}(\mathrm{SNR})$ via Monte Carlo simulations.

An SNR-dependent threshold in a similar context was first used by the TAMA group [5] who performed SNR-V /dof studies to veto out candidate events in their inspiral wave searches. See also [6] for a detailed description of a $\chi^{2}$ time-frequency test. In a context of a resonant bar detector burst search, see [7].

\section{Application}

To determine the false dismissal rate, the false alarm rate and the threshold equation for the veto statistic, we have performed a set of Monte Carlo simulations on artificial and real noise. We have used $10 \mathrm{~h}$ of fake Gaussian stationary noise and of real science data from the LIGO Hanford $4 \mathrm{~km}$ interferometer. The results presented here are thus valid for a $10 \mathrm{~h}$ observation time, which is the observation time of the all-sky, wide-band search that we plan to conduct on data from the second science run of the LIGO detectors. We do not take into account spin down of pulsars. This may be justified for the short time length of the data.

As will be explained below, we have injected both signals and spurious noise artefacts of the type that we observe in the detector output. The parameters of the gravitational wave signals which are injected into the noise are uniformly chosen at random in the following ranges: $f_{s} \in[100,500] \mathrm{Hz}, \alpha_{s} \in[0,2 \pi], \sin \delta_{s} \in[-1,1], \cos \iota \in[-1,1], \psi \in[-\pi / 2, \pi / 2]$, $\Phi_{0} \in[0,2 \pi]$. The strain $h_{0}$ or the amplitude of the model noise line is also randomly chosen in such a way that the resulting detection statistics value lie in the range: $\sqrt{50} \leqslant \sqrt{2 F_{\max }} \leqslant 70$. Below $2 F=50$ the efficiency of the test quickly degrades. We will thus not apply this veto 
technique to candidate events with $2 F<50$. In this sense, our method is designed to only discard large outliers.

\subsection{Safety test}

3.1.1. Signals in random Gaussian stationary noise. We have performed $2 \times 10^{6}$ Monte Carlo simulations. The following steps were executed iteratively 200 times:

- We randomly choose a signal frequency $f_{s}$ of a simulated gravitational wave and then follow the steps below 100 times: we randomly choose a signal sky position $\vec{l}_{s}=\left(\alpha_{s}, \delta_{s}\right)$ and perform the steps below 100 times:

1. We randomly choose a set of $\vec{p}_{s}$ signal parameters and generate the $10 \mathrm{~h}$ long data set described above consisting of random Gaussian noise and the fake signal.

2. We randomly displace the sky position template from the signal values by adding a random number uniformly distributed between \pm half the sky positions grid spacing: $\left|\alpha-\alpha_{s}\right| \leqslant 0.01$ and $\left|\delta-\delta_{s}\right| \leqslant 0.01$ (both in radians). The grid spacing was estimated numerically and ensures that the loss in $F$ due to the signal-template mismatch is less than $5 \%$ for $99 \%$ of the simulations, for a $10 \mathrm{~h}$ observation.

3. We search for the signal with template values $\vec{l}$ in a small frequency range around $f_{s}$. This results in the identification of an event, defined by a value of $F$ (the highest value of all the $F$ values in the event, denoted by $F_{\max }$ ) occurring at a frequency $f_{\max }$. Based on this we determine the maximum likelihood estimators $\vec{p}_{\text {MLE. Also, }}$ we compute $X_{i}\left(f_{k}, \vec{l}\right)$ for all the frequencies of the event.

4. We generate a veto signal $\tilde{s}\left(t ; \vec{p}_{\mathrm{MLE}}, \vec{l}, f_{\max }\right)$.

5. We compute $S_{i}\left(f_{k}, \vec{l}\right)$ for all the frequencies of the event.

6. We construct $\mathcal{V}$ from $\tilde{X}_{i}\left(f_{k}, \vec{l}\right)=X_{i}\left(f_{k}, \vec{l}\right)-S_{i}\left(f_{k}, \vec{l}\right)$.

By considering only values of $\sqrt{50} \leqslant \sqrt{2 F} \leqslant 70$, we obtain 1426915 sets of $F$ and $\mathcal{V}$ values. Figure 1 is the scatter plot of these. It is convenient to normalize each $\mathcal{V}$ by the corresponding number of degrees of freedom, dof, since dof could differ from one injection to another since the number of frequency bins in a candidate varies from event to event. If $\mathcal{V}$ follows the $\chi_{\text {dof }}^{2}$ distribution, then the mean of $\mathcal{V}$ is just dof. Figure 2 shows the estimated probability distributions of $\tilde{\mathcal{V}} \equiv \sqrt{\mathcal{V} / \text { dof }}$ for four selected ranges of $F$. These four graphs show that the probability distributions are well defined and the Monte Carlo simulations give a good estimate of the probability distribution of $\tilde{\mathcal{V}}$. Since a variable mismatch exists between the signals and the templates, the distribution of $\mathcal{V}$ is actually not strictly a central $\chi_{\text {dof }}^{2}$ and the expected value of $\tilde{\mathcal{V}}$ is thus not strictly 1 . And, as expected, the peak of the distributions of figure 2 deviates from 1 more as the signal becomes larger.

From figure 1 one can now define the threshold on the veto statistic, based on the false dismissal rate that one is willing to accept. The solid line in the figure, with equation

$$
\sqrt{2 F_{\max }}=10 \sqrt{\frac{\mathcal{V}}{\operatorname{dof}}}-10 \quad\left(7 \times 10^{-7} \text { false dismissal }\right),
$$

is the line with the lowest false alarm rate for which, with our sample size, we have not falsely dismissed any of the injected signals. In the rest of this paper, we will adopt this line, equation (7), as the nominal threshold line.

3.1.2. Signals in real data-LHO $10 \mathrm{~h}$. We have performed $2 \times 10^{6}$ Monte Carlo simulations by injecting a simulated pulsar signal into real data. All the steps are similar to those 


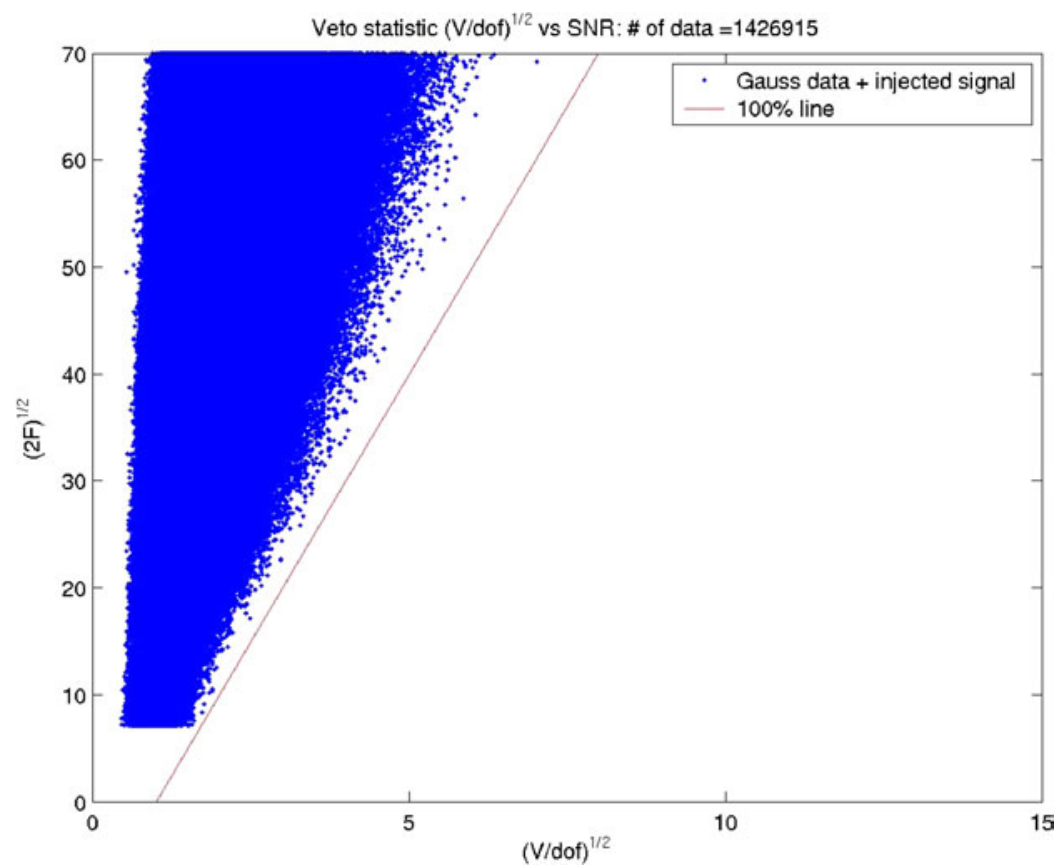

Figure 1. The veto statistic and the signal-to-noise ratio. This plot is for the $10 \mathrm{~h}$ simulated data. The detector is assumed to be the LHO detector. The number of data points with $\sqrt{50} \leqslant$ SNR $\leqslant 70$ is 1426915 .
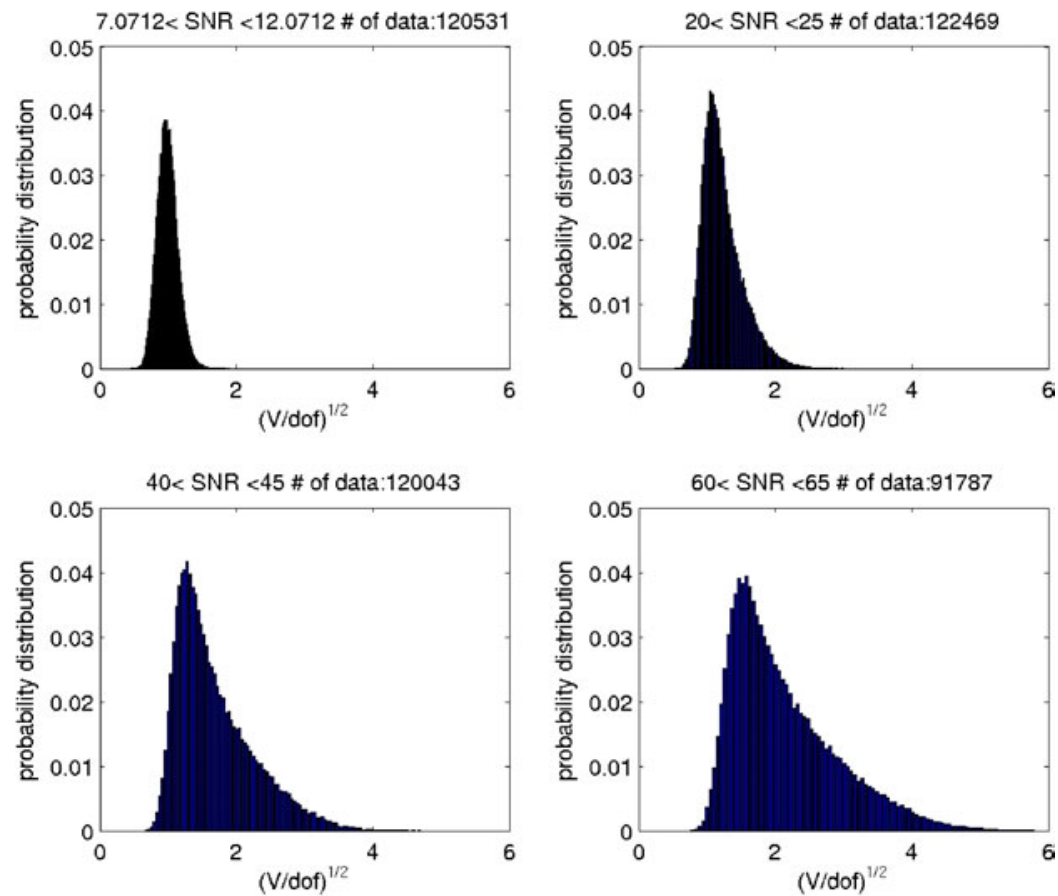

Figure 2. The estimated probability distributions for $\tilde{\mathcal{V}}$ with SNR in the four selected ranges. This figure is for the $10 \mathrm{~h}$ simulated data. The detector is assumed to be the LHO detector. 
described in section 3.1.1. We have avoided injections in bands contaminated by spectral disturbances.

From this set of simulations, we obtain a similar scatter plot as figure 1. And indeed, the threshold line equation (7) still does not dismiss any injected signals.

\subsection{Efficiency test}

To study how efficient the test is in vetoing noise artefacts that resemble the signals that we are trying to detect, we have performed an additional set of simulations. For each simulation, we have injected sets of time-domain exponentially damped sinusoids (as a model of a line noise) into both fake Gaussian noise and into real data. In the frequency domain these damped sinusoids have a Cauchy distribution, components of which are often observed in the real data. We hence follow similar steps as for the safety tests described above and produce the corresponding scatter plots of SNR versus $\tilde{\mathcal{V}}$.

The efficiency test here is ill defined in the sense that it is possible to generate infinite numbers of line noises that have completely different shapes from pulsar signals. Nonetheless, we think these tests provide 'a feel' for the efficiency of our veto method.

3.2.1. Noise lines in random Gaussian stationary noise. The following steps are iteratively performed 200 times:

- We randomly choose the frequency of a noise line and follow the steps below 100 times: we randomly choose a target sky position, with uniform distribution in $\alpha \in[0,2 \pi]$, $\sin \delta \in[-1,1]$ and then perform the steps below 50 times:

1. We randomly choose the noise line parameters. The e-fold decay rate varies between $0.01 / T_{0}$ and $2 / T_{0}$, where $T_{0}$ is the total observation time.

2. We generate a $10 \mathrm{~h}$ long data set consisting of random Gaussian noise with standard deviation 1 and the noise line defined by the parameters above.

3. We perform a search in a frequency band around the frequency of the noise line and identify an event, i.e., a value of $F$ and $f_{\max }$. From the values of the complex component of the detection statistic at $f_{\max }$ we determine $\vec{p}_{\text {MLE }}$ and $X_{i}\left(f_{k}, \vec{l}\right)$ for every frequency of the event.

4. We generate a veto signal $\tilde{s}\left(t ; \vec{p}_{\mathrm{MLE}}, \vec{l}, f_{\max }\right)$.

5. We compute $S_{i}\left(f_{k}, \vec{l}\right)$ for the veto signal for all the frequencies of the event.

6. We obtain $\tilde{\mathcal{V}}$.

Figure 3 shows the SNR- $\tilde{\mathcal{V}}$ plot. It may seem that the data points are densely distributed in the left upper region with large SNRs and small $\tilde{\mathcal{V}}$. This deceptive appearance is due to the coarse graphical resolution of the figure. This can be clearly seen in the estimated probability distributions shown in figure 4. In fact, if we take our nominal threshold line, equation (7), shown as the solid line in figure 3 , the false alarm rate is estimated to be $8.4 \%$.

3.2.2. Real data: $\mathrm{LHO} 10 \mathrm{~h}$. We have performed $10^{6}$ Monte Carlo simulations injecting noise lines as described above into real data, avoiding frequency bands with large noise artefacts.

The resulting scatter plot is similar to that obtained for the Gaussian random noise case. Indeed, we obtain $5.1 \%$ false alarm rate for the nominal threshold (equation (7)). 


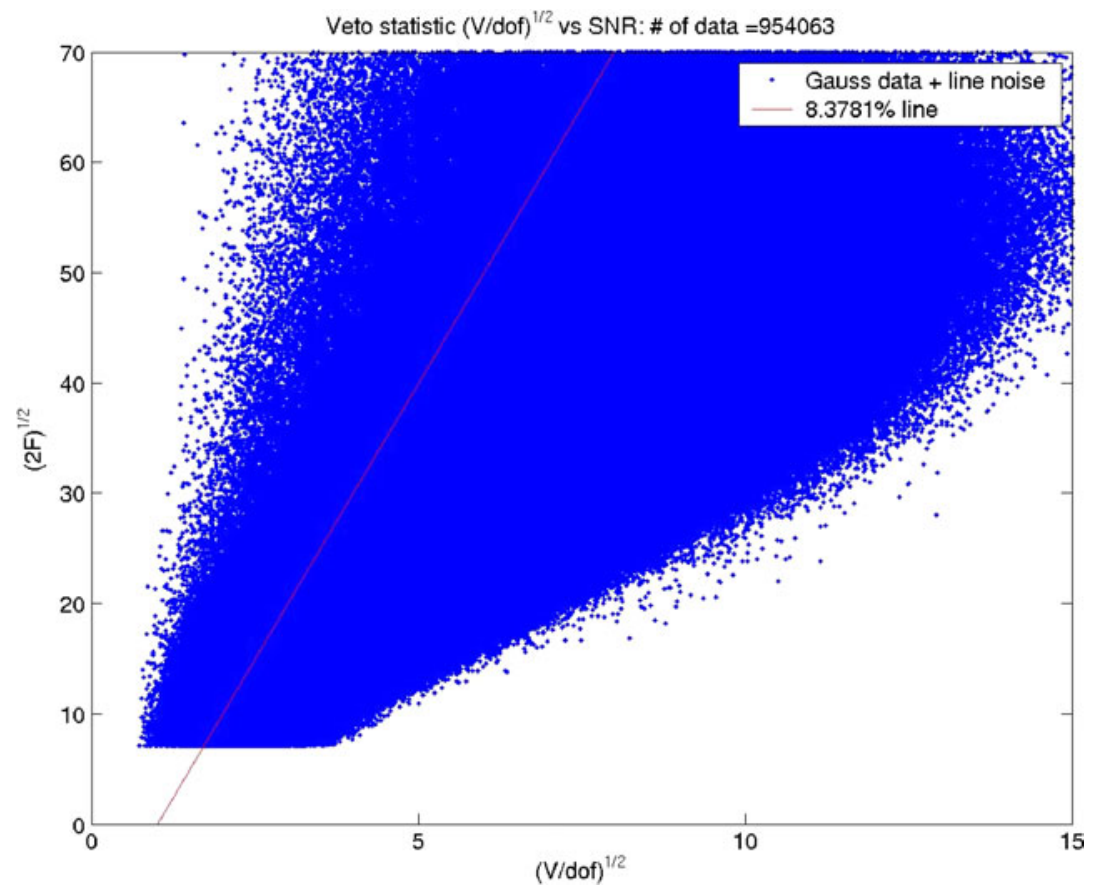

Figure 3. The veto statistic and the signal-to-noise ratio. This plot is for the $10 \mathrm{~h}$ simulated data. The detector is assumed to be the LHO detector. The number of data points with $\sqrt{50} \leqslant$ SNR $\leqslant 70$ is 954063 .
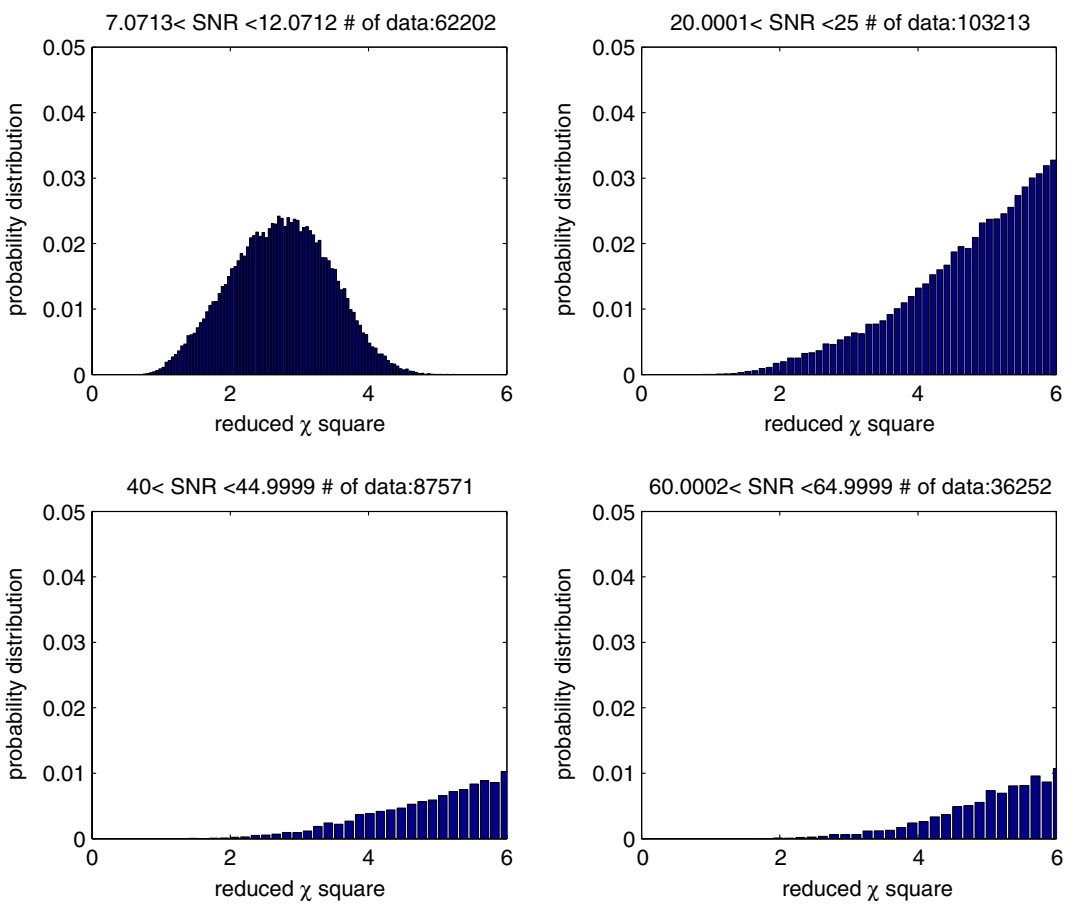

Figure 4. The estimated probability distributions for $\tilde{\mathcal{V}}$ with SNR in the four selected ranges corresponding to figure 3 . 


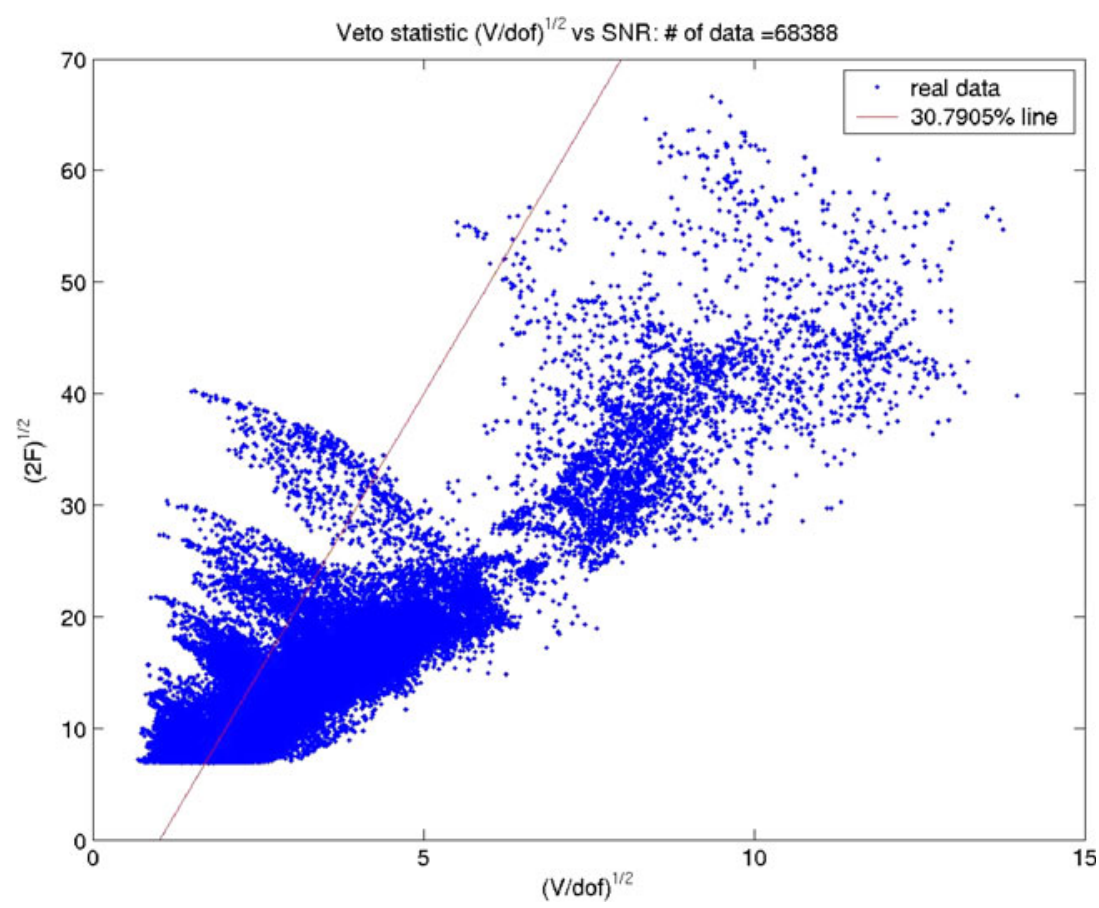

Figure 5. The veto statistic and the signal-to-noise ratio. This plot is for the $10 \mathrm{~h}$ real data of the LHO detector. The number of data points is 68388 . The maximum $\sqrt{2 F_{\max }}$ is 66.6.

\subsection{Application to real data}

Having observed safety and efficiency of our veto method, we now show an application of the method to real data (neither signal nor noise lines injected).

We take the following steps iteratively 1200 times:

- We randomly choose a template sky direction $\vec{l}$ over the whole sky.

1. We perform a wide-band search over the interval $[100,500] \mathrm{Hz}$ in the $10 \mathrm{~h}$ real data set. We identify events in the detection statistic and to each of these events we apply our veto test. This procedure yields a value of $F$ and $\tilde{\mathcal{V}}$ for each candidate event.

The scatter plot SNR $-\tilde{\mathcal{V}}$ is shown in figure 5. Two distinct branches along the solid line at higher SNRs are evident. Both branches are due to spectral features in the data: the highest SNR branch to a line at $465.7 \mathrm{~Hz}$, the lower branch to a line at $128.0 \mathrm{~Hz}$. These spectral features 'trigger-off' a whole set of templates giving rise to the observed structure in the scatter plot. If we adopt the threshold line (equation (7)), $70 \%$ of the events are rejected.

\section{Discussions}

We have defined a veto statistic to reject or accept candidate CW events based on a consistency shape test of the measured detection statistic. We have shown how to derive the SNR-dependent threshold for the veto test, through Monte Carlo simulations on a playground data set similar to the one that one intends to analyse.

The veto method demonstrated in this paper does not require any a priori information on the source of noise lines. However, we expect that the effectiveness of this veto technique can 


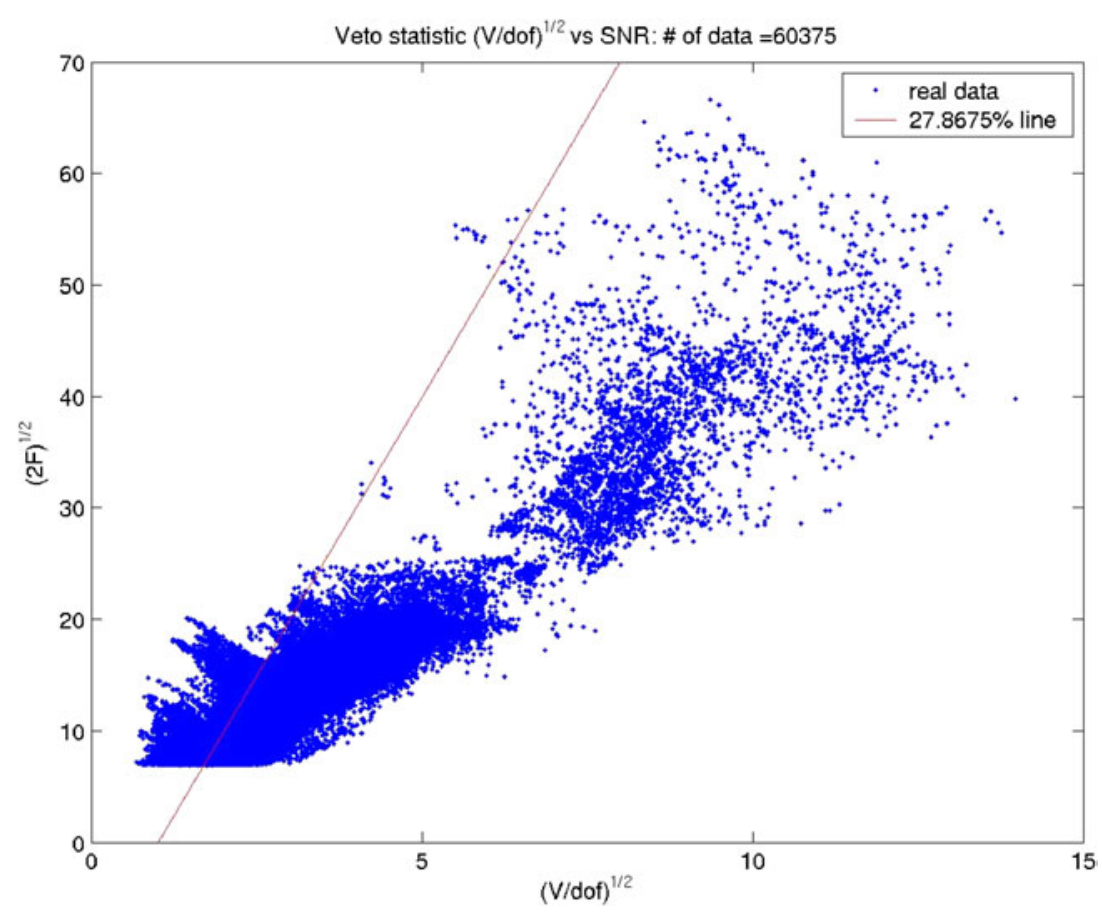

Figure 6. The same plot as shown in figure 5, but after removing the $16 \mathrm{~Hz}$ harmonics. The number of data points is 60375 . The maximum $\sqrt{2 F_{\max }}$ is 66.6.

greatly benefit from data characterization studies aimed at identifying spectral contamination of instrumental origin. We are now further investigating methods to veto out families of outliers identified in the scatter plots above the solid line in figure 5. Natural candidates are those noise lines whose properties are known experimentally, for example the $16 \mathrm{~Hz}$ harmonics in the LHO data due to the data acquisition system. It is precisely these harmonics that give rise to one of the major branches above the solid line in figure 5, as shown in figure 6.

In this paper, we have used a $10 \mathrm{~h}$ long data set. For a longer observational time, the difference between an extraterrestrial line and a terrestrial one becomes larger because the Doppler modulation patterns of a putative signal carry a more specific signature, that of the motion of the Earth around the Sun. We have not included spin down of pulsars in our current study, as we have used short enough time length data. Spin down effects of pulsars become more important for a longer observation time, and spin down effects generate a characteristic feature in the $F$ statistic shape. We thus expect that our veto method will become more efficient and safer for longer observation times.

Finally, we note that a veto threshold line varies depending on observational data time length and noise behaviour. The threshold line (equation (7)) is specifically for $10 \mathrm{~h} \mathrm{LHO}$ data, of particular band, and we recommend that any other search that uses quite different data set from our playground data should determine a threshold line based on playground data in each analysis.

\section{Acknowledgment}

We would like to thank M Landry, who has provided us with the tables of the experimentally measured noise lines of the data sets that we have analysed. 


\section{References}

[1] Jaranowski P, Królak A and Schutz B F 1998 Phys. Rev. D 58063001

[2] Astone P et al 2003 Class. Quantum Grav. 20 S665

[3] Abbott B et al (LIGO Scientific Collaboration) 2004 Phys. Rev. D 69082004

[4] Allen B et al 2004 Class. Quantum Grav. 21 S671

[5] Tagoshi H et al (TAMA Collaboration) 2001 Phys. Rev. D 63062001

[6] Allen B 2004 Preprint gr-qc/0405045

[7] Baggio L et al 2000 Phys. Rev. D 61102001 